\title{
INFLUENCE OF HEXAGONAL SYMMETRY STRESSES ON DOMAIN STRUCTURE AND MAGNETIZATION PROCESS $\mathrm{OF} \mathrm{FeBO}_{3}$ SINGLE CRYSTAL
}

\author{
Sharipov M.Z., Hayitov D.E., Raupova I.B., Sadikova M.I.
}

\section{Bukhara Engineering Technological Institute, Bukhara, Uzbekistan, m.Z.sharipov@rambler.ru}

\begin{abstract}
The magneto-optical method was used to study the effect of mechanical stresses on the domain structure, the magnetization process, and the main magnetic characteristics of the $\mathrm{FeBO}_{3}$ crystal. It is shown that the presence of mechanical stresses of $\mathrm{C}_{3}$ symmetry in the basal plane of the $\mathrm{FeBO}_{3}$ crystal leads to a rearrangement of its domain structure from 180 to 120 degrees, in which, unlike the usual domain structure, the spontaneous magnetization vector smoothly changes its azimuth in the basal plane along the direction domain boundaries. It was found that the appearance of a 120-degree domain structure leads to a substantial increase in magnetic anisotropy in the basal plane of a strained crystal, which leads to a change in its magnetic susceptibility and magnetization curve.
\end{abstract}

Keywords: single crystal, domain structure, magnetic field, temperature, thermodynamic theory, magneto-optics, mechanical stresses.

\section{Introduction}

The light-plastic weak ferromagnet, iron borate, is transparent in the visible region of the spectrum of electromagnetic waves, which, using magneto-optical methods, can conveniently and visually study the magnetic state and the magnetization process of this class of magnets. At temperatures below $T_{\mathrm{N}}$, a stable domain structure (DS) arises in $\mathrm{FeBO}_{3}$, which determines the process of technical magnetization of the crystal. In contrast to [1,2], where the experiments were carried out under conditions of uniform uniaxial crystal stress, the results of studies of the effect of inhomogeneous mechanical stresses on $\mathrm{FeBO}_{3} \mathrm{DS}$ are presented below. Transparent weak ferromagnets are (primarily due to the high mobility of domain walls) a promising medium for the elemental base of various devices of optical communication technology [3]; in addition to purely scientific interest, these studies were also stimulated by applied problems. Such problems inevitably arise when developing the design of one or another functional element of the device, when it is necessary to take into account possible changes in the main characteristics (magnetic susceptibility, coercive force, etc.) of the used magnetic material under the action of mechanical stresses caused, for example, by deformation of the attachment points of the functional element when the ambient temperature changes. The following are the results of studies of the effect of low-symmetric mechanical stresses on DWs and the magnetization process of this weak ferromagnet.

\section{Examples and experimental technique}

Samples of nominally pure (pure) iron borate single crystals were used as objects of study in the work. The synthesized crystals had the form of plates with a thickness of $\sim 50-70 \mu \mathrm{m}$ with transverse dimensions $\sim 3 \sim 5 \mathrm{~mm}^{2}$, the developed planes of which coincided with the basal (with the (111) plane). The crystals had surfaces of sufficiently high optical quality and did not require further polishing. For experiments, crystal samples were taken, the developed faces of which had a shape close to a regular hexagon. The orientation of the samples in the basal plane was carried out according to the natural faceting of the crystals. 
To create mechanical stresses in the crystal, the test sample was glued (BF-2 glue) to the copper washer in a predetermined manner (with its three angles) so that its center coincided with the center of the washer (Fig. 1), after which the washer with the sample was glued to the cryostat coolant. As the temperature decreases from room temperature, the washer deforms (its diameter decreases), which is transmitted to the sample, causing its elastic stresses. We do not know the coefficient of linear expansion of iron borate, however, as shown by our measurements, in this case the crystal is compressed in the basal plane along the radii of the washer.
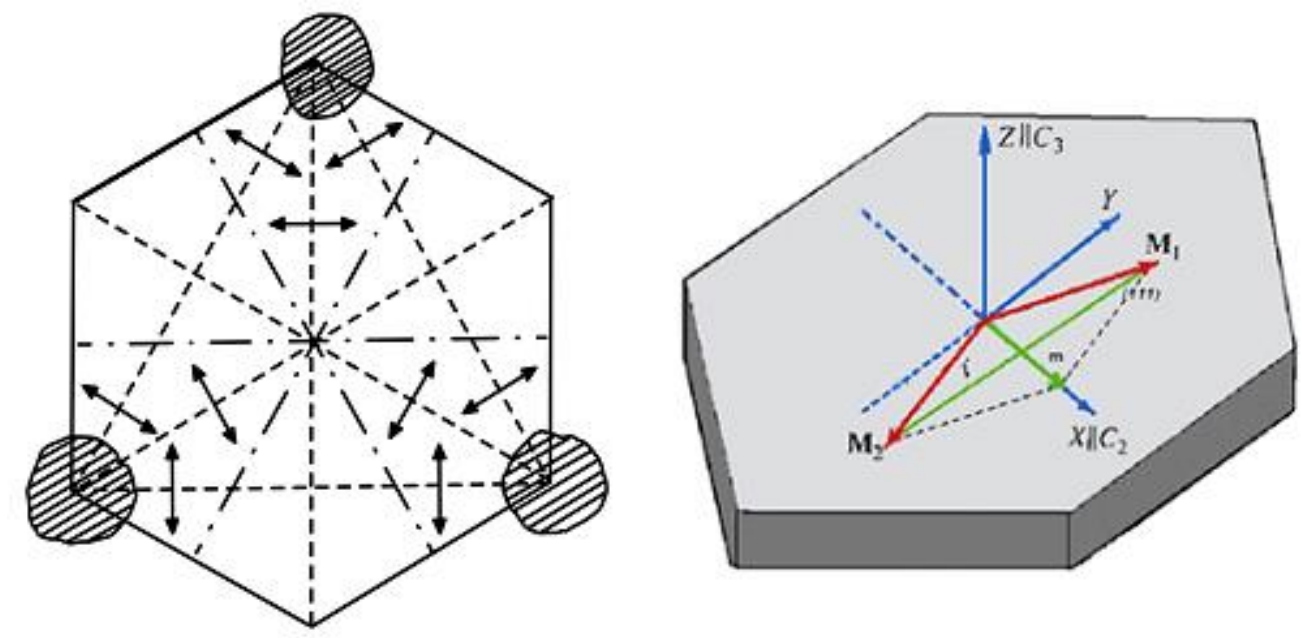

Fig.1. Schematic representation of the investigated sample: shaded areas - droplets of glue with which the crystal is glued to a copper washer; dashed lines - the main directions of emerging stresses; dash - dashed

lines - directions of $\mathrm{C}_{2}$ axes; double arrows indicate the preferred orientation of the vectors $\mathrm{m}$ in various regions of the crystal. On the right is the relative arrangement of the magnetizations of the $\vec{M}_{1}$ and $\vec{M}_{2}$ sublattices, as well as the vectors $\vec{m}=\vec{M}_{1}+\vec{M}_{2}$ and $\vec{l}=\vec{M}_{1}-\vec{M}_{2}$ in the basal plane of iron borate in the weakly ferromagnetic state.

The effect on the magnetic properties (domain structure, low-frequency magnetic susceptibility and magnetic hysterisis accompanying the process of technical magnetization) of the light-ferrous iron borate ferromagnet was studied under the influence of C3 symmetry stresses. The experimental results were carried out on an "unstressed" crystal and on a strained crystal, and also these results were compared with each other. Initially, the sample was placed in a mandrel of a sheet of thick paper about $10 \mu \mathrm{m}$ thick more than the thickness of the sample, in which a through hole was cut out according to the shape of the sample. The mandrel with the sample was glued around the perimeter between two $0.5 \mathrm{~mm}$ thick copper washers with central holes $1.5 \mathrm{~mm}$ in diameter, after which the whole structure was glued (using silicone vacuum grease) to the copper cold conductor of a nitrogen optical cryostat.

To study the effect of mechanical stresses on the magnetic state of iron borate, a magnetooptical technique for visualizing its DS was used. Images of domains were observed under a polarization microscope with an increase of $\sim 30-40 \times[4]$. The contrast of the obtained DS images arose due to different sizes and/or differences in the sign of the Faraday rotation angle for light transmitted through neighboring domains with different orientations of the vector $\mathbf{m}$. The domain structure of the sample and its evolution under the influence of a magnetic field and temperature was observed visually and recorded with a digital camera docked with a computer, or could be photographed using a photodetector. The process of technical magnetization was studied on the basis of hysteresis loops of the Faraday Effect that arose during magnetization reversal of a crystal in a quasistatic magnetic field that develops in time at a speed of $\approx 1 \mathrm{Oe} / \mathrm{s}$. 
Since the vector $\mathbf{m}$ lies in the plane of the sample and, therefore, the Faraday effect is possible only when the projection of the magnetization vector on the direction of light propagation appears, in all experiments the sample was oriented so that the normal to its plane formed an angle of $\sim 10^{\circ}$ with the direction of incident light.

\section{Discussion of experimental results}

The crystal symmetry of $\mathrm{FeBO}_{3}$ (space group $\mathrm{D}^{6}{ }_{3 \mathrm{~d}}$ ) allows the existence of 60,120 , and $180^{0}$ domain walls in the basal plane [5-12]. However, according to experiments [2, 3], temperature $180^{\circ}$ domain structure is realized in thin $\mathrm{FeBO}_{3}$ plates, which ensures a minimum of magnetoelastic energy. It's the main contribution to the thermodynamic potential of the crystal at $H=0$ [11]. Moreover, in stress-free crystals, domains form layers parallel to the basal plane along the thickness of the crystal with a Néel type domain boundary oriented along the $\mathrm{C}_{2}$ axes, which are $\mathrm{FeBO}_{3}$ easy magnetization axes (the domain boundary between domain layers is a Bloch type) [11]. As can be seen from Fig. 2a, just such a (two-layer) domain structure exists in the studied crystal at room temperature ${ }^{1}$. As the temperature decreases, the domain structure of the crystal continues to remain two-layer, however, the domain configuration gradually changes, acquiring at $T \sim 250 \mathrm{~K}$ the form shown in Fig. 2 b, which remains practically unchanged up to the minimum attainable in the experiment $T=90 \mathrm{~K}$.
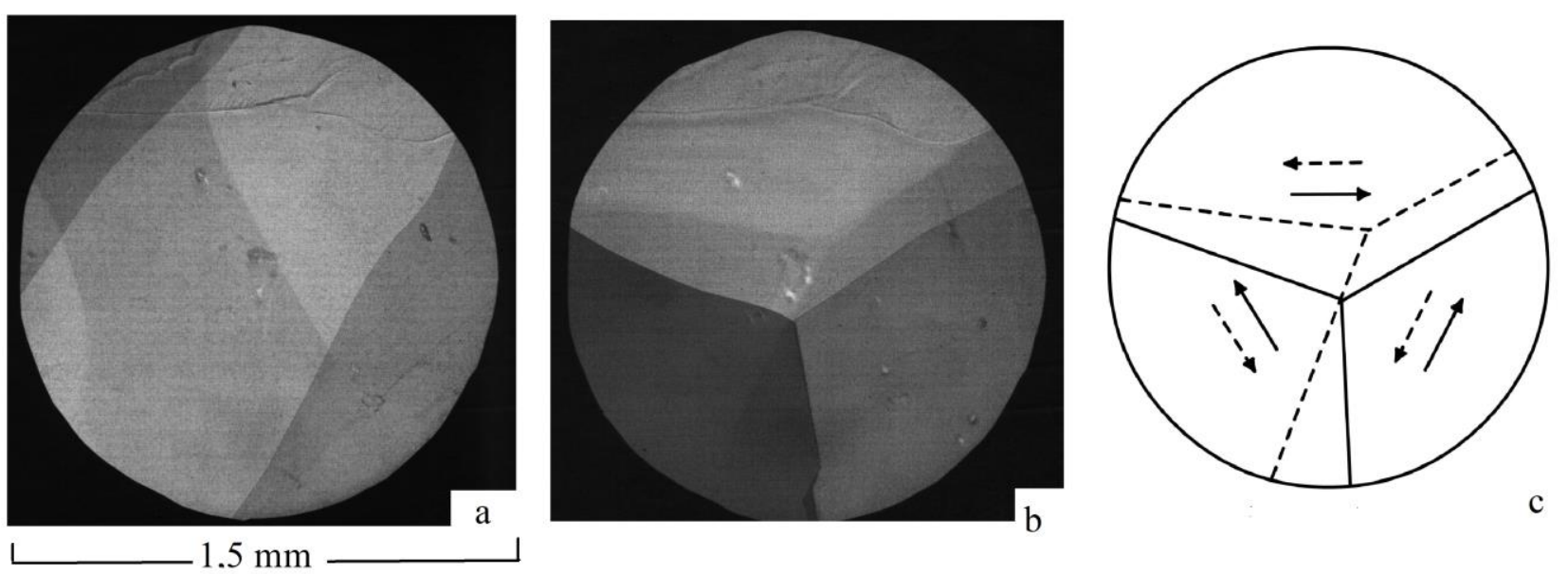

Fig. 2. The domain structure of a weak ferromagnet of iron borate obtained from the central part at a zero magnetic field: $\mathrm{a}-\mathrm{T}=295 \mathrm{~K}, \mathrm{~b}-\mathrm{T}=90 \mathrm{~K} ; \mathrm{c}$ - orientation geometry of the vector $\mathrm{m}$ in domains at $\mathrm{T}=90 \mathrm{~K}$.

The preferred orientation of the vector $\mathbf{m}$ in the crystal was determined by the reaction of the domain structure to an external magnetic field applied along different directions in the basal plane: with an increase in the field, the areas of domains in which the vector $\mathbf{m}$ makes an acute angle with the direction $\mathbf{H}$ grow due to domains with an energetically less favorable orientation $\mathbf{m}$ (Fig. $3 a, b$ ). The research results show that during compression of the light-weight ferromagnet of iron borate in the basal plane, the vector $m$ changes its orientation perpendicularly, and when stretched - parallel to the direction of stresses. In the stressed state, the spatial distribution of the ferromagnetism vector $\mathbf{m}$ in the domain structure is represented by the diagram in Fig. $2 c$. The resulting diagram shows that the iron borate sample is compressed, i.e. the linear expansion coefficient in $\mathrm{FeBO}_{3}$ is less than the linear expansion coefficient of copper in the entire studied temperature range.

Based on Fig. 26 , it can be concluded that the created stresses induce the so-called rotating anisotropy in a weak ferromagnet of iron borate, where the axis rotates in the basal plane. This leads

${ }^{1}$ All images of the domain structure presented in the work were obtained when the sample was rotated around the $Y$ axis by an angle of $\sim 10^{\circ}$ (the axes of the laboratory coordinate system are shown in Fig. 1). 
to a change in the boundary of its DS from $180^{\circ}$ to $\sim 120^{\circ}$ DS. In this case, each domain is divided by the thickness of the crystal into two layers with antiparallel vectors $m$ (due to the different thickness of the domain layers, the resulting Faraday rotation in each domain is nonzero, which ensures the magneto-optical contrast of the domain structure shown in Fig. 2). Note that, in contrast to the usual 120-degree domain structure, where the angle between the vectors $\mathrm{m}$ in neighboring domains is constant along the domain wall, in the observed domain structure of the "glued" sample due to the spatial heterogeneity of the arising stresses (see Fig. 1), the orientation of the vectors $m$ in both sides of the domain wall change along its length (it is obvious that the direction of the local vector $\mathrm{m}$ in the domains at $H=0$ is determined by the competition between the exchange and magnetoanisotropic interactions).
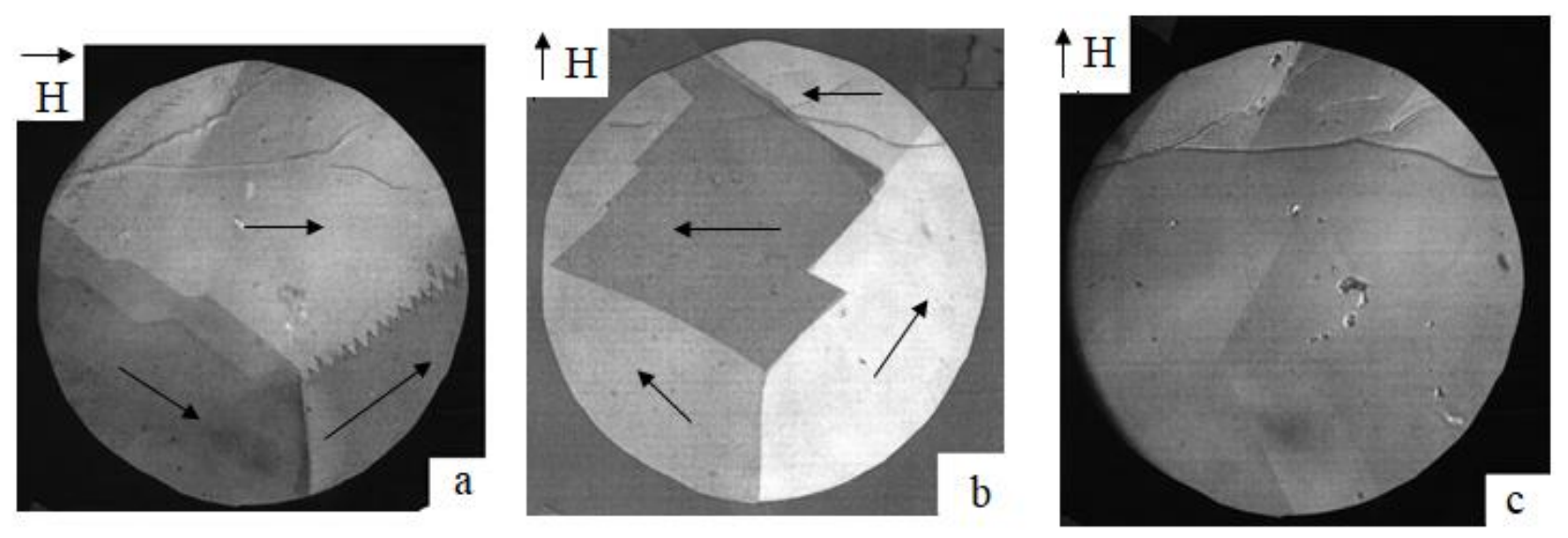

Fig. 3. Images of the central part domain structure of the stressed $\mathrm{FeBO}_{3}$ crystal obtained at $T=90 \mathrm{~K}$ : a) $-H=0.5$ Oe $(\vec{H} \| \vec{X})$, b) $-H=0.5$ Oe $(\vec{H} \perp \vec{X})$, c) $-H=1.5$ Oe $(\vec{H} \perp \vec{X})$. The arrows indicate the preferred direction of the vector $m$ in the domains; the arrow in the upper corner indicates the direction of magnetization.

The physical concepts of the magnetization of a crystal with domains prove that induced mechanical stresses increase its heterogeneity, and also cause an increase in the coercive force $H_{\mathrm{c}}$ [7], which we observed experimentally. As shown in fig. 4 at temperature $\mathrm{T}=90 \mathrm{~K}$ the field dependence $F(H)$, i.e. hysteresis loops of the "glued" sample are characterized by high coercivity. In the quasistatic mode of magnetic field sweep, the shape of the magnetic hysteresis loop changes depending on the direction of magnetization, as can be seen from Fig. 4, the dependence $F(H)$ of the "glued" sample, observed at $(\vec{H} \perp \vec{X})$, when reaching saturation, has an almost "hysteresisfree" quasilinear section that is absent in the analogous curve obtained at $(\vec{H} \| \vec{X})$.

To interpret the results of studies of the field dependence of the Faraday Effect in stressed $\mathrm{FeBO}_{3}$, we turn to Fig. 3, which shows the evolution of its domain structure in a weak magnetic field. From fig. 3 a, b that, when applying the field, first of all (due to the insignificant thickness of the sample) the boundaries between the domain layers disappear, as a result of which the domain structure becomes end-to-end with the orientation $\mathbf{m}$ in each domain, which corresponds to the most energetically favorable of the two possible at $H=0$ (see Fig. 3 a, b). Further growth of $H$ leads to a displacement of the domain wall in the basal plane of the crystal under the action of pressure $P$ [12]

$$
P=m H\left(\cos \theta_{1}-\cos \theta_{2}\right),
$$

where $\theta_{1}, \theta_{2}$ are the angles that the vector $\mathbf{m}$ makes up with $\mathbf{H}$ on both sides of the domain wall. 
As shown in Fig. $3 a, b$, with a perpendicular and parallel position of the magnetic field, one of the domain walls does not act on the magnetic field, due to the equality of angles $\theta_{1}$ and $\theta_{2}$, therefore, the magnetization of the crystal occurs mainly due to the displacement of two other domain walls. If we assume that the direction $\mathrm{m}$ in each domain does not change over the entire area of the domain, then substituting the values of the angles between the vectors $\mathbf{m}$ and $\mathbf{H}$ in (1), for the pressure acting on these domain walls, we obtain:

$$
\begin{aligned}
& \mid P\left(\vec{H}|| \vec{X}|=m H| \cos 0^{\circ}-\cos 60^{\circ} \mid=\frac{1}{2} m H,\right. \\
& \mid P\left(\vec{H} \perp \vec{X}|=m H| \cos 90^{\circ}-\cos 30^{\circ} \mid=\frac{\sqrt{3}}{2} m H,\right.
\end{aligned}
$$

then, ceteris paribus, to start an irreversible displacement of domain walls at $(\vec{H} \perp \vec{X})$ the field is required approximately 1.7 times less than at $(\vec{H} \| \vec{X})$. Taking into account the relation $H_{c} \propto \frac{1}{P}$ [9], from the last it follows:

$$
H_{c}(\vec{H} \| \vec{X}) \approx 1.7 H_{c}(\vec{H} \perp \vec{X}),
$$

which correlates with the experimental results (according to the graphs $F(H)$ presented in Fig. 4,

$$
\left.\frac{H_{c}(\vec{H} \| \vec{X})}{H_{c}(\vec{H} \perp \vec{X})} \approx 1.5\right) \text {. }
$$

The difference between the values of $H_{c}(\vec{H} \| \vec{X})$ and $H_{c}(\vec{H} \perp \vec{X})$, less pronounced in comparison with the experimentally derived from (1), can probably be attributed to the already mentioned change in the angle between the vectors $\mathbf{m}$ in neighboring domains along domain walls.

So, for example, from a comparison of Fig. 1 and Fig. $3 a, b$, it can be seen that in the middle part of the formed domain walls the angles between the vectors $\mathbf{m}$ on both sides of the domain walls are $\sim 180^{\circ}$, and therefore, in these sections of the domain walls, the ratio between $|P(\vec{H} \| \vec{X})|$ and $|P(\vec{H} \perp \vec{X})|$ it turns out to be the opposite of what was obtained above.

From fig. $3 a, b$, we can conclude that at the final stage of the process of displacement of domain walls (when domain walls disappear and the crystal transitions to a single domain state) at $(\vec{H} \| \vec{X})$, the vector $\mathbf{m}$ over the entire area of the sample is approximately parallel to $\mathbf{H}$, while at $(\vec{H} \perp \vec{X})$ the vectors $\mathbf{m}$ and $\mathbf{H}$ form a sharp angle between themselves. In the latter case, the further magnetization of the crystal, obviously, occurs by turning the vector $\mathbf{m}$ towards $\mathbf{H}$. This causes the appearance of a "hysteresis-free" quasilinear region on the $F(H)$ curve observed during magnetization of strained $\mathrm{FeBO}_{3}$ along the $\mathrm{Y}$ axis (Fig. 4). Fig. $3 \mathrm{c}$ illustrates the magnetic state of the sample at this stage of the magnetization process.

It is known that, at a sufficiently fast change in the value of magnetization of iron borate in the basal plane occurs exclusively by rotation vector $\mathbf{m}$. Based on this fact, it can be assumed that in our experiments when the magnetization is reversed, the sample $m$ in the alternating magnetic field with a frequency of $v=95 \mathrm{~Hz}$ rotates the vector $\mathbf{m}$ in the domains toward $\mathbf{H}$. 


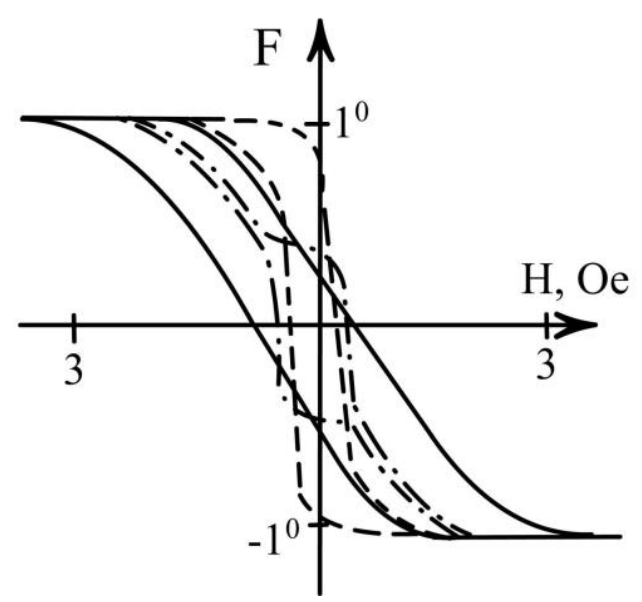

Fig.4. Field dependence of the Faraday Effect in $\mathrm{FeBO}_{3}$. The dashed line is the $F(H)$ dependence for the "unstressed" crystal, the solid and dashed lines are the $F(H)$ dependences for the "glued" sample, observed at $T=90 \mathrm{~K}$ with the field orientation $(\vec{H} \| \vec{X})$ and $(\vec{H} \perp \vec{X})$, respectively.

Therefore, the shape of the dynamic hysteresis loop is practically independent (in contrast from the $F(H)$ curves observed under the quasistatic magnetization regime) from the orientation of $\mathbf{H}$ in the basal plane of the crystal.

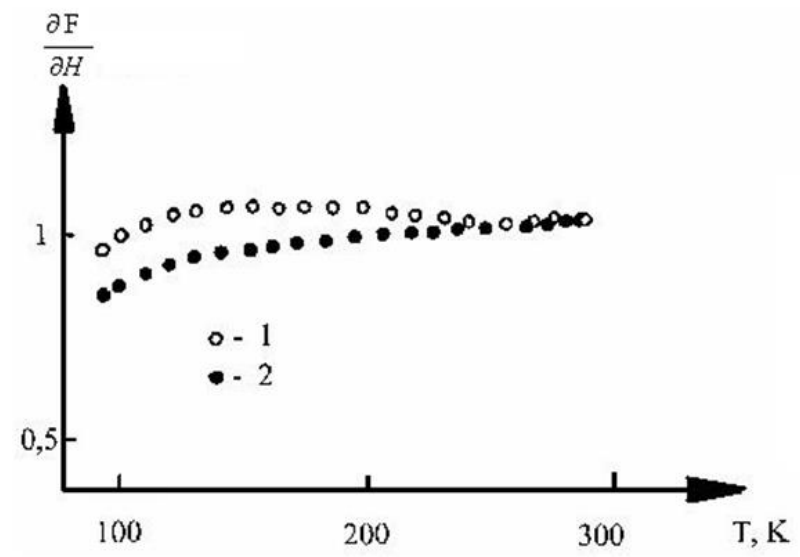

Fig. 5. Temperature dependence of the magneto-optical susceptibility of $\mathrm{FeBO}_{3}$ :

1 - "glued" sample, 2- "unstressed" crystal.

From the graphs presented in Fig. 5, it can be seen that at $\mathrm{T}<250 \mathrm{~K}$ the value $\frac{\partial F}{\partial H}$ for the "unstressed" crystal decreases monotonically with decreasing temperature, while for the "glued" sample the $\frac{\partial F}{\partial H(T)}$ dependence has a more complex form. It was established that the initial magnetic susceptibility $\chi$ in $\mathrm{FeBO}_{3}$ is determined by the reversible shift of the Bloch domain walls separating the domains along the thickness of the crystal (therefore, the anisotropy of $\chi$, and hence the anisotropy of the $\frac{\partial F}{\partial H}$ quantity, is absent in the basal plane of the crystal).

In this case $\chi \propto \frac{m^{2}}{K}$ 


$$
\text { ( } K=\frac{H_{a}}{M_{s}}-\text { in-plane anisotropy constant). From the known data on the dependences } m(T)
$$

and $K(T)$, it follows that at $\mathrm{T}<290 \mathrm{~K}$, with decreasing temperature, $m^{2}$ increases more slowly than the constant $K$, which determines the form of the dependence of the $\frac{\partial F}{\partial H(T)}$ "unstressed" crystal (Fig. 5).

Turning to Fig. 1, one can notice that in the central part of the crystal the magnitude of the arising stresses is relatively small, and the directions of the stress vectors are least defined (the radius of "curvature" of the anisotropy axis is maximum). If we do not take into account the exchange interactions, it follows from the latter that in the central part of the "glued" sample all possible orientations $\mathbf{m}$ in the basal plane are approximately equivalent from the energy point of view. Obviously, this circumstance facilitates the rotation of the vector $\mathbf{m}$ under the action of the field in the basal plane of the crystal. Consequently, in stressed $\mathrm{FeBO}_{3}$, the process of the displacement of the Bloch domain boundary along its thickness begins in a weaker magnetic field, which is likely to determine a certain increase in the magneto-optical susceptibility of the "glued" sample (Fig. 5) ${ }^{1}$

\section{Conclusion}

It is shown that the presence of mechanical stresses of $\mathrm{C}_{3}$ symmetry in the basal plane of the $\mathrm{FeBO}_{3}$ crystal leads to a rearrangement of its domain structure from 180 to 120 degrees, in which, unlike the usual domain structure, the spontaneous magnetization vector smoothly changes its azimuth in the basal plane along the direction domain boundaries.

It was found that the appearance of a 120-degree domain structure leads to a substantial increase in magnetic anisotropy in the basal plane of a stressed crystal, which leads to a change in its magnetic susceptibility and magnetization curve.

\section{Acknowledgments}

The authors are grateful to doctor of phys.-math. sci., prof. Sokolov B.Yu, doctor of phys.-math. sci., prof. Djuraev D.R. and prof. Zhumaev M.R. for the discussion of the results. The work was carried out with the financial support of the Ministry of Innovation Development of the Republic of Uzbekistan in the framework of project OT- $\$ 2-64$ "Fluctuation and parametric phenomena in condensed and nanoscale systems".

\section{REFERENCES}

1 Bogdanova Kh.G., Golenishchev-Kutuzov V.A., Medvedev L.I., et al. Weak distortions of the magnetic structure of iron borate. Sov. Phys. JETP. 1989, Vol. 68, No. 2. pp. 345-349.

2 Lacklison D.E., Chadwick J., Page J.L. Photomagnetic effect in ferric borate. J. Phys. D: Appl. Phys. 1972, Vol. 5, pp. $810-821$.

3 Scott G.B. Magnetic domain properties of $\mathrm{FeBO}_{3}$. J. Phys. D: Appl. Phys. 1974, Vol. 7, No.11, pp. $1574-1587$.

4 Boidedaev S.R., Dzhuraev D.R., Sokolov B.Yu., Sharipov M.Z. Magnetooptical method of investigation of the magnetic inhomogeneity of easy-plane antiferromagnets with a weak ferromagnetism. Optics and Spectroscopy, 2008, Vol. 104, No. 4, pp. 604-609.

\footnotetext{
${ }^{1}$ Considering the heterogeneity of the crystal stresses, in this case it is more correct to speak not about the displacement of the Bloch domain wall in the field $\mathrm{H}$ as a whole, but about its bending, similar to the deformation of a flat elastic membrane under the action of a force applied normally to the plane of the membrane in the center of the membrane.
} 
5 Sharipov M.Z., Mirzhonova N.N., Hayitov D.E. Effect of inhomogeneous radially directed mechanical stresses on the domain structure of a $\mathrm{FeBO}_{3}$ single crystal. Eurasian Physical Technical Journal, 2019, Vol.16, No.1(31), pp. $35-41$.

6 Sharipov M.Z., Dzhuraev D.R., Sokolov B.Yu., Kurbanov M. Modulated magnetic structure of an inhomogeneously stressed single crystal $\mathrm{FeBO}_{3}$. Ukr. J. Phys. 2010. Vol. 55, No. 6. pp. $706-711$.

7 Sokolov B.Yu., Sharipov M.Z. Influence of the biaxial mechanical stress on the domain structure of holmium yttrium garnet ferrite. Russian Physics Journal. 2014, Vol. 57, No 8, pp. $1001-1007$.

8 Sharipov M.Z., Hayitov D.E., Rizoqulov M.N., Islomov U.N., Raupova I.B. Domain structure and magnetic properties of terbium ferrite-garnet in the vicinity of the magnetic compensation point. Eurasian Physical Technical Journal. 2019. Vol. 16, No. 2(32), pp. $21-25$.

9 Sokolov, B.Y., Sharipov, M.Z. Magneto-optical properties of the iron garnet $\mathrm{Tb}_{3} \mathrm{Fe}_{5} \mathrm{O}_{12}$ near the magnetic compensation temperature. Physics of the Solid State, 2014, Vol.56, No. 5, pp. 975 - 979.

10 Sokolov B.Yu., Sharipov M.Z. Low - frequency resonance of domain walls in the iron garnet $\mathrm{Tb}_{3} \mathrm{Fe}_{5} \mathrm{O}_{12}$ near the magnetic compensation point. Journal of Experimental and Theoretical Physics. 2013, Vol. 116. No 5. pp. 775-779.

11 Farztdinov M.M. Physics of magnetic domains in antiferromagnets and ferrites. Moscow, Nauka, 1981, 156 p. [in Russian]

12 Tikadzumi S. Physics of ferromagnetism. Moscow, Mir, 1987, 420 p. [in Russian].

Article accepted for publication 28.04 .2020 